IdeAs

Idées d'Amériques

$12 \mid 2018$

Le tourisme dans les Amériques

\title{
Le tourisme dans la Caraïbe, un moteur du développement territorial
}

El turismo en el Caribe, un motor del desarrollo territorial

Tourism in the Caribbean, a Strong Factor of Territorial Development

Michel Desse, Jusline Rodne Jeanty, Monique Gherardi et Simon Charrier

\section{CpenEdition}

Journals

Édition électronique

URL : https://journals.openedition.org/ideas/4239

DOI : $10.4000 /$ ideas.4239

ISSN : 1950-5701

Éditeur

Institut des Amériques

Référence électronique

Michel Desse, Jusline Rodne Jeanty, Monique Gherardi et Simon Charrier, « Le tourisme dans la

Caraïbe, un moteur du développement territorial », IdeAs [En ligne], 12 | 2018, mis en ligne le 21

novembre 2018, consulté le 18 octobre 2022. URL : http://journals.openedition.org/ideas/4239 ; DOI : https://doi.org/10.4000/ideas.4239

Ce document a été généré automatiquement le 18 octobre 2022

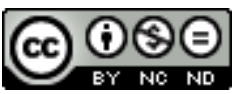

Creative Commons - Attribution - Pas d'Utilisation Commerciale - Pas de Modification 4.0 International - CC BY-NC-ND 4.0

https://creativecommons.org/licenses/by-nc-nd/4.0/ 


\section{Le tourisme dans la Caraibe, un moteur du développement territorial}

El turismo en el Caribe, un motor del desarrollo territorial

Tourism in the Caribbean, a Strong Factor of Territorial Development

Michel Desse, Jusline Rodne Jeanty, Monique Gherardi et Simon Charrier

Cette recherche a bénéficié de l'appui du programme de recherche collectif C3AF (Changement Climatique et Conséquences sur les Antilles Françaises), co-financé par la Région Guadeloupe et l'Union européenne (programme FEDER)

\section{Introduction}

1 Les îles de la Caraïbe développent leur économie touristique depuis les années 1930 et surtout depuis les années 1970. Elles constituaient alors les destinations tropicales privilégiées, mais leur poids a diminué aujourd'hui : en 2015, 25 millions de touristes internationaux se sont rendus dans la Caraibe, sur un total de 199,3 millions pour l'ensemble des Amériques, et de 309 millions pour l'Asie et le Pacifique (UNWTO, 2017). La concurrence des destinations tropicales asiatiques (32,6 millions de touristes internationaux pour la Thaïlande en 2016) est donc forte. Néanmoins, les îles de la Caraïbe constituent un bassin touristique dynamique et en constante évolution, avec 11,4 millions de touristes internationaux en 1990 et 25, 2 en 2015, irrigué par la clientèle nord-américaine et par les flux européens qui se portent chacun vers leurs anciennes possessions.

2 L'activité touristique a succédé à l'agriculture d'exportation; le club de vacances et le grand hôtel ont remplacé le rôle économique et social de la distillerie ou de l'usine sucrière (Desse M., 2003, 2004, 2013). Ce passage d'un système à un autre s'achève dans certaines stations touristiques des petites îles qui ont renforcé leur tertiairisation par les activités financières off shore. D'autres espaces amorcent ce changement. Les 
situations sont donc multiples et engendrent une juxtaposition des formes de tourisme : de séjour, de croisière, de plaisance, de grande plaisance, de niveau d'accueil et d'équipement, du luxe à l'équitable.

3 Ce développement est aujourd'hui accentue par l'intérêt porté par les habitants aux pratiques ludo-sportives balnéaires et nautiques (Desse M., 2005, 2006). L'activité touristique apparaît comme un moteur dynamique du développement territorial insulaire, offrant toute la palette des possibles et s'adressant aux clientèles locales et régionales des pays de niveau intermédiaire, aux touristes issus de l'émigration et aux touristes internationaux les plus exigeants. Enfin, le secteur touristique s'adapte aussi à l'évolution régressive des littoraux, permettant de diminuer l'habitat de bord de mer et d'accroître les nouvelles fonctionnalités touristiques que s'approprient alors les habitants.

Après avoir rappelé le contexte de la mise en tourisme et la diffusion spatiale, largement inféodée au niveau du développement, nous aborderons deux exemples : la croisière et la grande plaisance. Il s'agit de deux secteurs bien différents en termes de flux touristiques et d'infrastructures, mais dans les deux cas les acteurs insulaires montrent un grand dynamisme pour capter l'ensemble de ces flux. Les clientèles sont différentes, mais les stratégies mises en place montrent une forte complémentarité. Enfin, à l'échelle de la plage, du village de pêcheur s'ouvrant au tourisme, nous montrerons comment dans certains cas, comme à Haïti, l'enclave et son système ségrégatif demeurent, alors qu'en Guadeloupe une plus grande ouverture est de mise, permettant des retombées à un secteur plus important de la population.

\section{Un contexte dynamique et une forte diffusion spatiale}

5 Si les Grandes Antilles ont connu une mise en tourisme relativement ancienne, dès le début $\mathrm{du} \mathrm{XX}^{\mathrm{e}}$ siècle, dans la continuité du développement touristique en Floride (Miossec J-M., 1998), les Petites Antilles connaissent un développement plus tardif de l'activité touristique. Trinidad et la Barbade se sont ouvertes dans les années 1960, alors que les Antilles françaises, Antigua, Sainte-Lucie et les îles hollandaises ne deviennent des destinations importantes que dans les années 1970 et surtout depuis 1980. La Dominique et Saint-Vincent restent encore à l'écart des flux touristiques et demeurent des destinations à découvrir (tableau 1).

Tableau 1- Les îles qui accueillent plus de 400000 touristes de 1980 à 2006

Sources : Carribean Tourism Organization 


\begin{tabular}{|l|l|}
\hline 1980 & $\begin{array}{l}\text { Bahamas, Bermudes, Îles Vierges américaines, Jamaïque, } \\
\text { Porto Rico, }\end{array}$ \\
\hline 1990 & $\begin{array}{l}\text { Bahamas, Barbade, Bermudes, Îles Vierges américaines, } \\
\text { Jamaïqu, Porto Rico, République dominicaine, Sint } \\
\text { Maarten }\end{array}$ \\
\hline 2000 & $\begin{array}{l}\text { Aruba, Bahamas, Barbade, Bermudes, Cuba, } \\
\text { Guadeloupe, Îles Vierges américaines, Jamaïque, } \\
\text { Martinique, Porto Rico, République Dominicaine, Sint } \\
\text { Maarten }\end{array}$ \\
$\begin{array}{l}\text { Aruba, Bahamas, Barbade, Bermudes, Caïmans, Cuba, } \\
\text { Guadeloupe, Îles Vierges américaines, Jamaïque, } \\
\text { Martinique, Porto Rico, République dominicaine, } \\
\text { Trinidad et Tobago, Sint Maarten }\end{array}$ \\
\hline
\end{tabular}

Dans toutes les îles, le développement du tourisme s'appuie sur une forte implication des pouvoirs publics. À la Barbade, l'Hotel Aids Act signé en 1956 est à l'origine du développement touristique (Burac M., 1993). On compte déjà 16 hôtels en 1957, 175 en 1982, 165 en 1987 et près de 150 actuellement. La Barbade a connu son plus haut niveau de construction d'infrastructures en 1982, avec 14314 lits.

7 En Guadeloupe, les différents plans entre 1956 et 1980, qui s'intègrent dans les politiques globales de rattrapage économique, encouragent la construction de grands hôtels afin de diversifier l'économie guadeloupéenne. En 1956, la société ministérielle SITO (Société immobilière et touristique d'Outre-Mer), créée pour l'ensemble des DOM, développe la grande hôtellerie et les infrastructures de base, comme le réseau routier, l'aéroport, les réseaux d'eau et d'électricité. Entre 1961 et 1965, le IV plan propose ainsi la construction d'hôtels de luxe d'une capacité de 500 chambres, l'amélioration des infrastructures d'équipement et la formation du personnel. Le financement était en partie assuré par des fonds publics. En 1965, une mission interministérielle est créée afin de regrouper des équipements structurants (casino, ports de plaisance, practices de golf) et d'éviter ainsi l'isolement des grands hôtels (Larroque-Chounet L.,1989). Le Gosier, Sainte-Anne et Saint-François deviennent des stations touristiques dotées des grands équipements structurants comme les ports de plaisance à Saint-François et à Gosier, le golf et le casino à Saint-François, les clubs de vacances et les grands hôtels dans chaque station. Diverses incitations financières et fiscales sont octroyées aux investisseurs hôteliers, attirant ainsi les capitaux métropolitains. Au milieu des années 1970, les plans envisagent toujours l'essor de la grande hôtellerie, mais aussi le renforcement de petites et moyennes structures afin d'ouvrir le secteur touristique aux capitaux insulaires. Depuis les années 1980, les aides se portent sur la mise à niveau des infrastructures portuaires et aéroportuaires, sur les réseaux routiers et toujours sur l'hôtellerie, mais aussi sur les gîtes, les locations saisonnières, les restaurants, les 
structures d'animation et la location de bateaux de plaisance (Chardon J-P, Hartog T., 1995).

Depuis 1986, l'État met en place des régimes d'aide fiscale en faveur des investissements productifs dont le tourisme (Desse M., 2000, Dehoorne O., 2007). Au milieu des années 1960, la capacité hôtelière atteint 500 chambres, 837 en 1970, 3038 en 1980, 4740 en 1988 et 6000 chambres en hôtels, résidences de tourisme et villages de vacances en 2002. La Guadeloupe n'est ainsi entrée dans une phase de touristification qu'à la fin des années 1970.

Certaines îles ont connu une mise en tourisme plus tardive. Durant les années 1980 et 1990, des îles comme Trinidad, Aruba, Anguilla qui avaient parié sur le développement industriel et pétrolier, sont confrontées à des crises économiques et se lancent dans le tourisme pour diversifier leurs économies. Les grosses unités hôtelières se développent à Aruba après la fermeture de la raffinerie pétrolière Exxon en 1985; on compte aujourd'hui 7500 chambres (Desse M., 2013). Dans la même logique, l'État crée la compagnie Air Aruba pour dynamiser son tourisme en ouvrant des liaisons directes avec les Amériques du Nord et du Sud. À Sainte-Lucie, depuis 1996, l'État s'engage sur la voie du développement touristique par des aménagements structurants, la mise à niveau des réseaux routiers, la construction du terminal de croisière de la Pointe Séraphine, la réhabilitation de la place à charbon dans le centre-ville de Castries, les retenues d'eau douce afin d'alimenter l'hôtellerie (Desse M., 2005, 2007). Enfin, le Saint Lucia Tourist Board, institution qui dépend du ministère du Tourisme, se charge des campagnes publicitaires diffusées sur les écrans canadiens, américains et britanniques, et dans les salons internationaux. Le gouvernement intervient enfin auprès des populations locales afin de les sensibiliser à l'accueil et à la nécessité économique de cette activité.

Ainsi à la fin des années 1990, toutes les îles connaissent une augmentation notable du nombre de touristes. La fréquentation touristique de la République dominicaine passe ainsi, entre 1992 et 2000, de 1,8 million à 2,9 millions de touristes, puis à 4,4 millions en 2006, les îles Vierges anglaises de 100000 à 281000 puis à 544000 en 2006. Seule l'île de Montserrat, en partie évacuée et dévastée par l'éruption de son volcan depuis 1997, a connu une forte diminution de sa fréquentation.

Dès la fin des années 1990, on note la relative stagnation de certaines destinations, qui s'explique par la concurrence des pays asiatiques, le vieillissement du parc hôtelier des premières îles comme la Barbade, la Martinique, les Bahamas et les Antilles néerlandaises.

Au contraire, les nouveaux complexes de Punta Cana à Saint-Domingue ou de Cayo Coco à Cuba attirent et se situent dans leur phase ascendante, liée à la découverte de la destination. À Cuba, où l'économie souffre de l'embargo américain et de la perte de ses acheteurs attitrés en Europe de l'Est depuis 1989, le tourisme apparaît comme un moyen de diversifier l'économie et de gagner des devises étrangères. On comptait 50000 touristes en 1975, 372000 en 1992, 1 million en 1996 et 1,8 millions en 2000 . Entre 1990 et 2001, la fréquentation touristique a progressé de $431 \%$. Les atouts sont nombreux : la variété des paysages, la richesse du patrimoine et de la musique, mais aussi la politique de l'État cubain qui ouvre ce secteur aux capitaux étrangers, surtout espagnols, par des joint-ventures, fait appel à des conseillers européens et crée la société étatique de gestion, la Cubanacan responsable de chaînes d'hôtels et de tours opérateurs. Les grands hôtels internationaux sont privilégiés pour développer le tourisme de masse (Desse M., 2013). Ce partenariat se poursuit, puisque 17 sociétés internationales exploitent 50 hôtels et permettent d'offrir près de 15600 chambres. 
11 Dans les années 2000, la fréquentation touristique des îles de la Caraïbe connaît un ralentissement, et cette tendance est renforcée dans les Antilles françaises. La crise mondiale marquée par les attentats aux États-Unis, l'augmentation des prix du pétrole, puis la crise des sub-primes affectent tout particulièrement ce secteur. D'après la Caribbean Tourism Organization, 17, 9 millions de touristes ont fréquenté les îles en 2008, soit une diminution de $6 \%$ par rapport à 2007. Si 35,3\% des touristes ont fréquenté la République dominicaine et Cuba ou les perles touristiques comme Saint-Kitts, les îles Vierges, Aruba ou Saint-Barthélemy... seuls 2,7\% des touristes ont séjourné à la Martinique. Ce déclin est particulièrement important pour le tourisme de croisière qui s'élevait à 87000 croisiéristes en 2008 contre 414000 en 1998. En Guadeloupe, la croisière a aussi connu une baisse de $75 \%$ de sa fréquentation (Desse M., 2017). Ainsi des cycles de fréquentation touristique apparaissent et signent le déclin des anciennes destinations, comme Saint-Martin, la Guadeloupe, la Martinique et la Barbade, au profit de nouvelles comme Cuba, la République dominicaine, Anguilla et Trinidad.

Depuis 2010, la région Caraibe est redevenue attractive avec 24 millions de touristes internationaux en 2015 (4 millions de plus depuis 2010). Les Grandes Antilles dominent toujours avec la République Dominicaine (5,6 millions), Cuba et Porto-Rico (3,5 millions), La Jamaïque (2,1 millions). Barbade, Saint-Martin, la Guadeloupe et la Martinique attirent 500000 touristes. En dessous, les flux peuvent être plus ténus, mais rapportés au nombre d'habitants et à la superficie, ils s'avèrent très importants et génèrent des revenus par habitant conséquents (cf. figure 1).

Figure 1 : Tourisme international et recette associée dans les Caraïbes en 2015

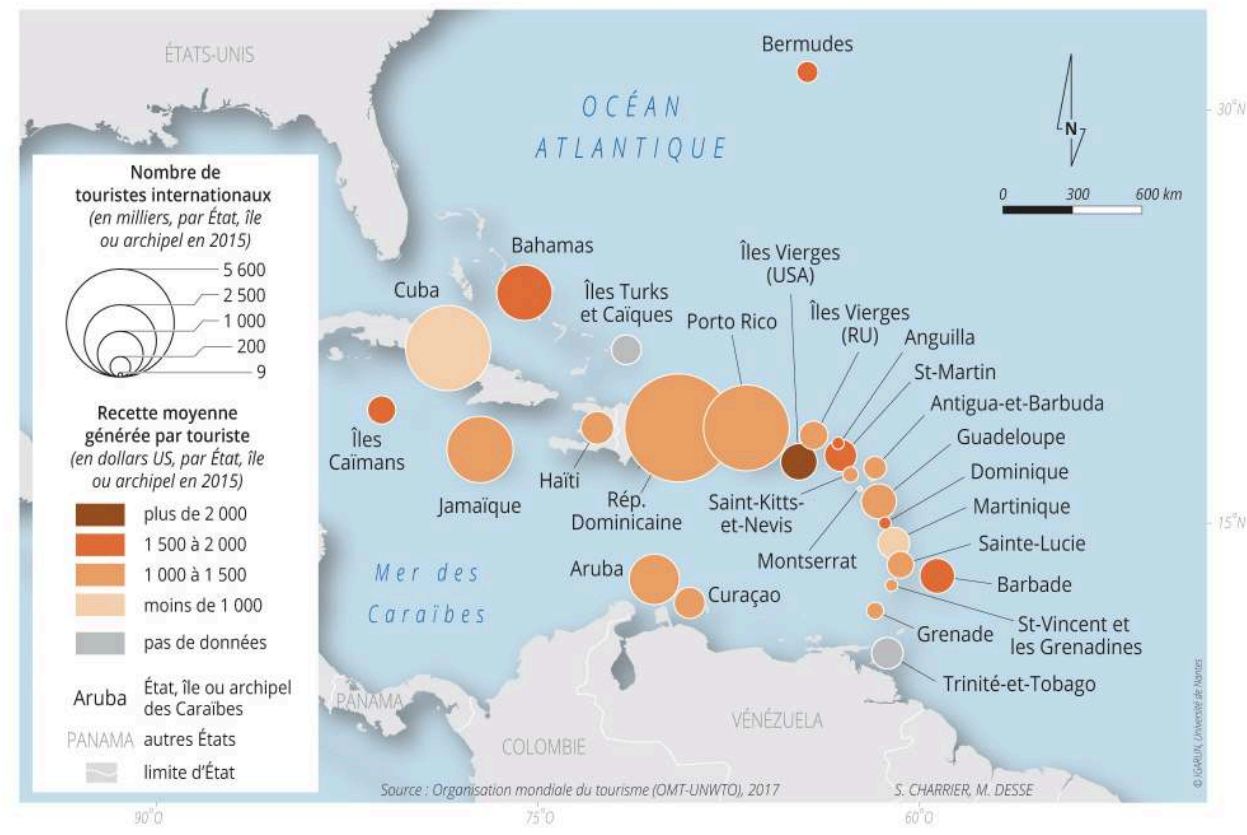




\section{S'adapter aux formes du tourisme: l'exemple de la croisière et de la grande plaisance}

13 Alors que le tourisme a été initié par des plans de développement structuraux et la mainmise de grands opérateurs internationaux, dès que les niveaux de vie se sont améliorés, les initiatives insulaires se sont développées, du petit vendeur du secteur informel à l'hôtellerie de luxe à base de capitaux insulaires, en passant par toutes les déclinaisons du tourisme durable. Depuis les années 1960, les acteurs insulaires s'adaptent aux marchés porteurs, aux nouvelles attentes ou à certaines niches touristiques prometteuses. C'est le cas du tourisme de croisière qui constitue une activité ancienne dans la Caraïbe, et de la grande plaisance qui apparaît dans son ampleur comme un secteur touristique en forte progression.

\section{Accueillir paquebots et croisiéristes}

14 Si la progression du nombre de croisiéristes semble régulière, cela n'empêche pas une forte rivalité et des évolutions locales notables qui ont un impact sur les économies touristiques. La Caraïbe constitue toujours la première région de croisières, regroupant 33,7 \% de l'offre, loin devant la Méditerranée (18,7\%).

15 Dans le bassin caribéen, Miami domine l'ensemble et assure le transit de près de 10 millions de passagers. Les autres ports importants en position de «tête de ligne » sont: Port Canaveral, Tampa, Fort Lauderdale et San Juan de Puerto Rico. Les destinations les plus fréquentées restent les îles du nord de l'archipel, ainsi que les rivages du golfe du Mexique, la Nouvelle-Orléans, Cancun ou Nassau (Bahamas), qui accueillent 2,4 millions de passagers). Six destinations rassemblent les deux tiers des croisiéristes : les Bahamas et les îles Vierges américaines (13\%), Cozumel au Mexique (11\%), Porto Rico (9\%), les îles Caïmans $(8 \%)$ et les îles néerlandaises, principalement Sint Maarten (8\%). À l'opposé, Saint-Vincent et les Grenadines accueillent 124000 croisiéristes, et Trinidad et Tobago 58 700. Les autres îles des Petites Antilles accueillent entre 100000 (Saint Kitts et Nevis) et 616000 à Barbade (Desse M., 2017).

16 Si dans les années 1990 le nombre de ports capables d'accueillir des paquebots était réduit, les fortes retombées économiques ont engendré une âpre concurrence entre les îles qui se sont ouvertes à cette activité (Desse M., 1996, 2001). Les destinations anglophones ont su se moderniser et attirer la clientèle nord-américaine (Connel J., 1998). Au contraire, Pointe-à-Pitre et Fort-de-France ont souffert de la concurrence, et nécessitent des adaptations importantes (Dehoorne O et Petit-Charles N., 2011). Alors qu'en 1996, la Guadeloupe recevait 589500 croisiéristes, la fréquentation n'a cessé de décroître à 292000 en 2000 et seulement 77000 en 2007, soit une baisse de $75 \%$. On ne comptait que 12300 passagers de paquebots en escales et seulement 64700 croisiéristes partant de Pointe-à-Pitre, qui est aussi un port tête de ligne malgré les faillites de la compagnie Festival et les difficultés de Nouvelles Frontières et de la Compagnie des îles du Ponant. L'activité connaît à nouveau une reprise avec 111000 croisiéristes en 2010, 158354 en 2013, 320000 croisiéristes en 2016 et 400000 en 2017. 


\section{Des aménagements portuaires importants}

17 Dans toutes les îles des Petites Antilles, pendant la haute saison (de décembre à avril), ce sont trois à cinq paquebots qui accostent par jour et par escale, délivrant plusieurs milliers de croisiéristes qui deviennent alors majoritaires dans les petites villes. Avec la modernisation et l'extension des zones portuaires depuis les années 1960, de nombreuses villes se sont coupées de la mer (Desse M., 1996). Dans ces conditions, la rupture était très forte pour les croisiéristes qui quittaient leur décor luxueux pour débarquer sur un quai bordé d'entrepôts et de dents creuses urbaines... Cependant, devant les retombées économiques que peuvent apporter quelques milliers de croisiéristes chaque jour pendant plusieurs mois, les municipalités ont, dès les années 1990, rivalisé d'efforts pour ouvrir des corridors permettant de rejoindre le centre historique, pour construire des terminaux dédiés à la croisière et pour aménager les fronts de mer. Il s'agissait à l'époque de rendre les villes attractives aux compagnies de croisière, dans un contexte de vive concurrence interinsulaire. Pointe-à-Pitre, Fort-deFrance et Castries ont en effet perdu le charme de leurs maisons en bois, des petits métiers, des marchés, et constituaient à la fin des années 1980 de véritables repoussoirs pour les activités touristiques, cantonnées aux plages et à quelques promenades en forêt. Depuis 1988 en Guadeloupe et 1991 à la Martinique, les municipalités de Pointe-àPitre et de Fort-de-France valorisent les fronts de mer, à l'instar d'autres escales comme Civitavecchia, Barcelone et ses 9 terminaux de croisière, ou les travaux actuels à Miami qui s'achèveront en 2035 (Desse M., 2013, 2017).

18 À Fort-de-France, certains paquebots accostent encore dans le port de commerce, qui, excentré, impose l'utilisation de taxis pour rejoindre le centre-ville. Cependant, d'autres accostent depuis 1998 au ponton de la Pointe Simon long de 270 mètres. Le front de mer a été complétement remodelé depuis 2010, avec, d'une part, les tours modernes de la Pointe Simon et, d'autre part, un aménagement paysagé donnant sur une petite plage au pied du Fort Saint-Louis. Un marché créole est implanté au débouché du ponton d'arrivée des croisiéristes. Le centre-ville et ses commerces se modifient lentement par une mise en couleur des façades. Les restaurants, les magasins de mode et de souvenirs remplacent les vieux comptoirs portuaires. Le Grand Port Maritime de la Martinique réaménage aussi le terminal croisière des Tourelles dans le port de commerce afin de soutenir le développement des têtes de ligne permettant l'accueil de deux paquebots. Un vaste espace est reconfiguré pour assurer l'avitaillement et la sécurisation du site et pour devenir une zone d'animation (terrain de sport, mini-golf), où les passagers pourront se divertir et se restaurer. Une petite gare maritime sera également installée pour permettre aux croisiéristes d'embarquer directement sur des bateaux afin de visiter les petites îles et la zone hôtelière de la Pointe du Bout, située sur la rive sud de la Baie de Fort-de-France. Tout ceci correspond au grand essor que connaît à nouveau la croisière dans les Antilles françaises (Desse M., 2017).

Image 1 : Le Grand Port Maritime de la Martinique vante sa logistique (pouvoir accueillir 5 paquebots par jour) et montre ainsi que Fort-de-France est redevenue une escale attractive.

Sur l'image du bas, un paquebot accoste à la Pointe Simon, nouvelle vitrine moderne de la ville. En arrière-plan, 4 navires de plus petite taille sont à quai au terminal de croisière des Tourelles dans le port de commerce, plus excentré.

Source : Le Marin, 20 septembre 2018. 


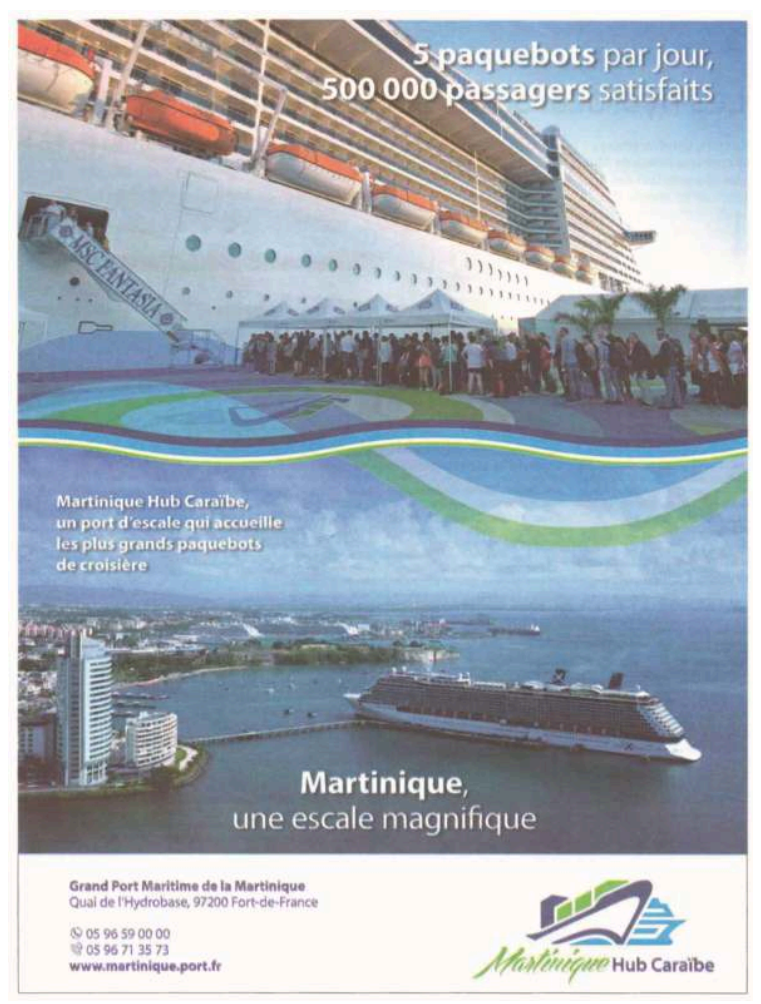

Les politiques urbaines qui ont mené à la réhabilitation des fronts de mer ont été très dirigistes, avec pour but essentiel d'attirer les paquebots pour en capter des ressources financières. La mise en tourisme de ces quartiers s'accompagne chaque fois d'une métamorphose de l'appareil commercial: reconstruction du marché de Castries, affectation des vieilles halles de Roseau au profit de l'artisanat local (Mario S., 2010). Les hangars de bord de mer sont réaffectés en restaurants, galeries d'art, magasins de souvenirs. Dans les Antilles françaises, les parfumeries, les bijouteries, les magasins de mode ponctuent discrètement les quelques rues arpentées par les croisiéristes. À la Pointe Séraphine à Castries, comme au centre Saint-John Perse à Pointe-à-Pitre, une galerie marchande parfois détaxée offre tous les produits internationaux, à quelques pas de la passerelle d'embarquement. Ces quartiers de la croisière sont aussi des lieux de mixité et sont appropriés par les insulaires, qui surtout en basse saison constituent l'essentiel de la clientèle (Desse M., 2007).

\section{La Grande plaisance, une nouvelle niche en voie d'appropriation...}

Avec la Méditerranée, la Caraïbe constitue un important bassin de croisière, qui offre de nombreux atouts : des îles nombreuses permettant des trajets de courte durée, des petites îles discrètes et bien équipées, un grand nombre de centres financiers offshore permettant l'immatriculation des pavillons de complaisance, la forte implantation d'entreprises de refit (entretiens et hivernage des navires) et la présence d'un grand nombre de richissimes propriétaires et locataires.

21 Longtemps réservée à une petite élite, souvent liée à l'économie maritime, la grande plaisance tend à se développer en nombre d'unités, mais aussi par les dimensions des grands yachts. En 2016, d'après le Global Order Book, les propriétaires sont originaires des États-Unis, d'Italie, d'Allemagne et de Grande-Bretagne. Viennent ensuite la Russie et le Moyen-Orient. $80 \%$ des yachts sont loués, il est donc rare qu'un yacht soit 
uniquement utilisé par son propriétaire. La location permet d'entretenir le navire et son équipage, et devient rentable avec 10 à 12 semaines de location. Les loyers peuvent atteindre des sommes très élevées en fonction du yacht et de son standing ... 750000 euros par semaine pour un navire de 60 mètres pour 12 passagers et 14 membres d'équipage. Enfin, certaines formules permettent de s'adapter à des segments de clientèle différents pour des événements ou des séjours festifs comme les yachtings days, ou les fêtes de Noël à Saint-Barthélemy (Desse M., Charrier S., 2017).

Figure 2 Les capacités d'accueil des yachts de plus de 25 mètres dans la Caraïbe

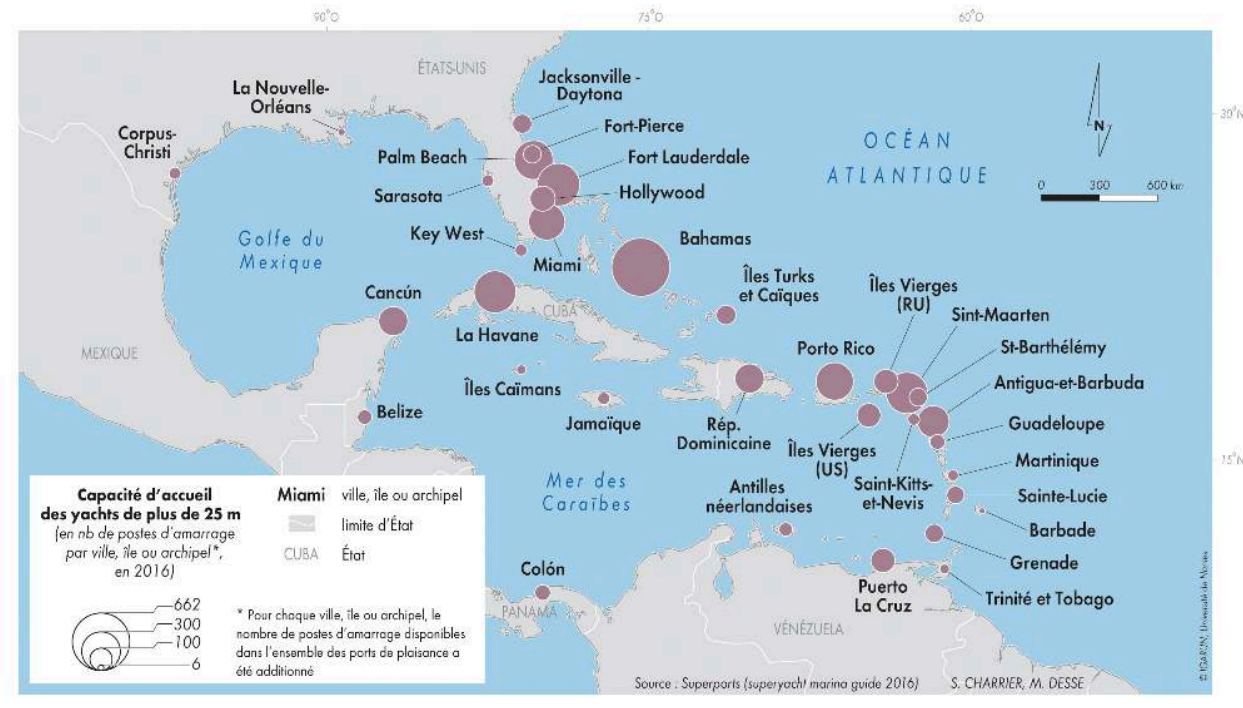

Dans ces conditions, les ports s'équipent d'aménagements très haut de gamme, entourés de complexes immobiliers et de commerces de luxe. Ces retombées idéelles et économiques expliquent les plus fortes concentrations en Floride et au Nord de l'Arc antillais. On compte, en 2016, 22 ports en Floride et 46 pour l'ensemble des îles de la Caraïbe. Le nord de la Caraïbe regroupe les plus fortes capacités d'accueil : 662 places pour les 15 ports de l'archipel des Bahamas, 347 places à Sint-Maarten (7 ports), 187 places à Antigua (5 ports). Au sud de l'arc antillais, le nombre de places est limité et ne concerne souvent qu'un unique port de plaisance : 40 places à Pointe à Pitre, 20 places au Marin, 6 à Grenade, 16 à Trinidad.

Les sorties en mer peuvent s'effectuer pour quelques heures au départ des différents ports de Floride, des Bahamas ou d'Antigua. Parfois les périples sont plus longs, à l'occasion d'évènements festifs ou de salons du yachting (Fort Lauderdale Boat Show, Antigua Yacht show). D'autres enfin iront d'île en île privilégiant les escales sécurisées aux îles Vierges, à Saint-Barthélemy et Saint-Martin, et le long des côtes sous-le-vent de Guadeloupe (Deshaies) ou de Martinique (Anses d'Arlet, Anse Mitan). Canouan ou les Tobago Kay dans les Grenadines constituent les destinations méridionales les plus avancées (Desse M., 2013). La haute saison (décembre à avril) est complémentaire avec celle de la Méditerranée. Dans tous ces ports, une économie du luxe s'est développée : depuis l'accueil VIP dans les aéroports pour les avions privés, la restauration, l'événementiel, la maintenance et la surveillance. Saint-Barthélemy, qui est une zone franche, attire ainsi un tourisme de luxe, des croisiéristes naviguant sur de petites ou moyennes unités de croisière (145 paquebots pour 48022 croisiéristes en 2016). Depuis quelques années, la grande plaisance investit les quais et constitue une part de plus en 
plus importante des passagers arrivés au port, avec plus de 7000 yachts pour 39485 passagers en 2016. Ces croisiéristes recherchent à Gustavia les produits détaxés haut de gamme, les restaurants de cuisine française - l'originalité d'une touche française dans un environnement anglophone (Desse M. et Nordin C., 2015).

\section{L'appropriation par les habitants : quel développement territorial ?}

D'une île à l'autre, en fonction des façades au-vent ou sous-le-vent, du dynamisme des acteurs, du niveau de développement, l'activité touristique ne crée pas les mêmes leviers de développement.

\section{Une difficile appropriation à Haïti}

Dans les régions enclavées marquées par un faible développement et parfois, comme à Haïti, par une grande pauvreté, le tourisme est peu approprié par les habitants et ne permet pas un véritable développement territorial. Malgré un développement touristique ancien, le tourisme, $\mathrm{du}$ fait du contexte politique, sanitaire et environnemental, demeure balbutiant à Haïti. Il reste confiné aux enclaves - les coquilles hôtelières -, qu'il s'agisse du loisir populaire sur les plages publiques, du tourisme des classes aisées et des expatriés, comme celui des quelques touristes internationaux, alors que les touristes issus de la diaspora haïtienne recherchent les hôtels moyens de gamme, qui demeurent néanmoins des enclaves sécurisées (Calmont A, Mérat P-J., 2015).

La plage de Labadie connaît une importante fréquentation et fonctionne comme une enclave, permettant aux excursionnistes des navires de croisière de profiter des plaisirs d'une plage tropicale : les parasols, le marché créole où l'on achète de l'art haïtien et la location d'engins de plage donnent les saveurs d'un tourisme de séjour. Cette enclave fixe sur la plage ces croisiéristes, alors que toute la région nord d'Haïti, riche en patrimoine historique et environnemental, pourrait profiter de cette manne financière. L'effet d'enclave se perçoit ici à travers l'absence des croisiéristes en dehors du site d'accueil. Alors que le village de Labadie se situe à proximité du site, il se trouve dans la même situation de pauvreté que le reste du territoire haïtien, puisque les croisiéristes ne peuvent quitter la plage, qui est une enclave ouverte seulement aux excursionnistes et fermée aux Haïtiens qui ne travaillent pas sur place.

Cette enclave de Labadie accueille les navires de la compagnie Royal Caribbean International depuis 1985 (Doura F., 2012), soit près de 781556 croisiéristes en 2017 pour 154 escales de navires selon les données de l'Autorité Portuaire Nationale du CapHaïtien. D'après les statistiques de la Société Labadie Nord (SOLANO), il y a eu en 2013 certaines retombées positives, puisque l'on décompte près de 700 emplois : 230 directs (mécaniciens, agents de sécurité, personnel d'entretien, cuisiniers, réceptionnistes, guides touristiques) et 470 environ interviennent de manière occasionnelle (commerçants, musiciens, danse folklorique). Les produits artisanaux proviennent essentiellement de la ville du Cap-Haïtien et de la zone frontalière avec la République dominicaine. L'État bénéficie aussi des retombées, en taxant de 2 dollars américains les 
différents vendeurs et depuis 2016, 12 dollars par croisiériste (pour 781556 excursionnistes et 154 navires en 2017) (Autorité Portuaire Nationale du Cap-Haïtien).

Sur la plage de Labadie, les deux mondes ne se côtoient pas, séparés par un grand mur entre le village et les croisiéristes. Cette forteresse est vue comme une prison pour les excursionnistes, surtout avec la présence soutenue des agents de sécurité. Comme il n'y a pas de restaurants haïtiens, les excursionnistes consomment uniquement les aliments préparés à bord du paquebot, ce qui constitue un manque à gagner pour la population de la région nord et pour les 2000 habitants de Labadie, toujours marqués par le sousemploi et la grande pauvreté.

Tableau 2- Arrivées des touristes au port d'escale de Labadie (2011- mars 2018)

Source : réalisé à partir des données de l'APN du Cap-Haïtien

\begin{tabular}{|l|l|l|l|l|l|l|l|l|}
\hline Année & $\mathbf{2 0 1 1}$ & $\mathbf{2 0 1 2}$ & $\mathbf{2 0 1 3}$ & $\mathbf{2 0 1 4}$ & $\mathbf{2 0 1 5}$ & $\mathbf{2 0 1 6}$ & $\mathbf{2 0 1 7}$ & $\begin{array}{l}\text { mars } \\
\mathbf{2 0 1 8}\end{array}$ \\
\hline Touristes & 169710 & 638303 & 779073 & 748667 & 672177 & 821707 & 781556 & 181075 \\
\hline Navires & 41 & 148 & 188 & 183 & 164 & 184 & 154 & 37 \\
\hline
\end{tabular}

Image 2 : Les installations de plage pour accueillir les croisiéristes : le décor est planté : eaux transparentes et calmes, sable blanc. Cette plage privée ne s'anime qu'avec l'arrivée des paquebots de croisière.

Source : Jusline Rodne/Jeanty, avril 2018

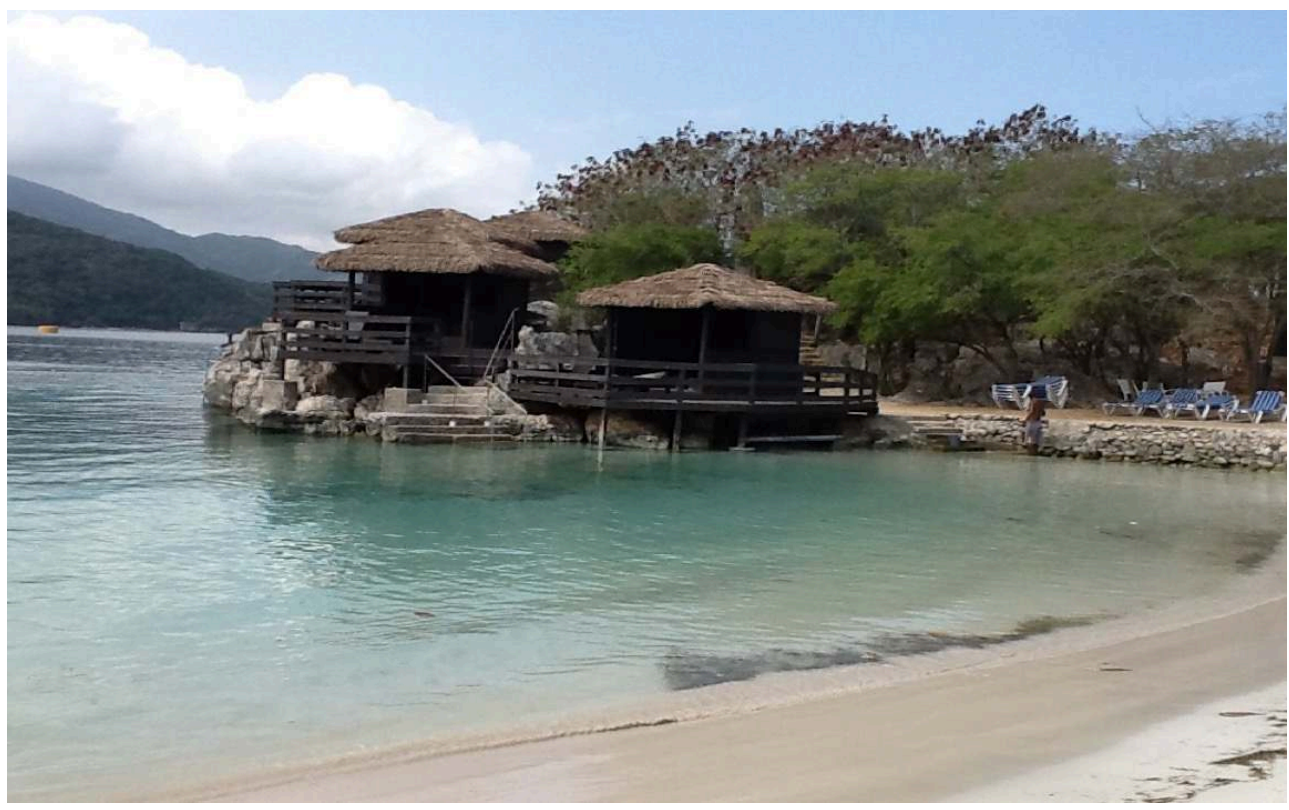

Image 3 : Commerce de produits artisanaux sur le site de Labadie

Source : Jusline Rodne/Jeanty, avril 2018 


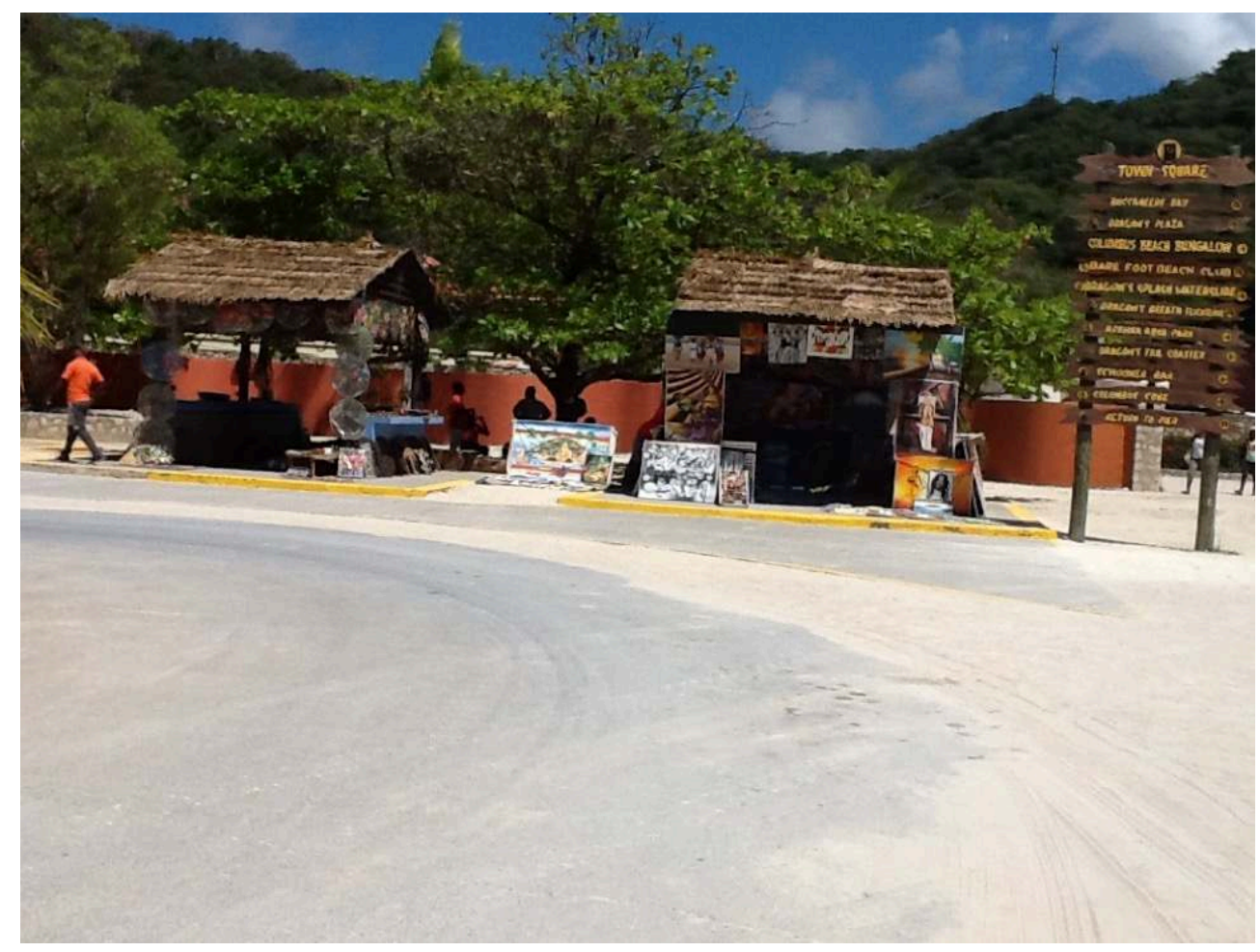

Image 4 : Animateur sur le site de Labadie

Source : Jusline Rodne/Jeanty, avril 2018

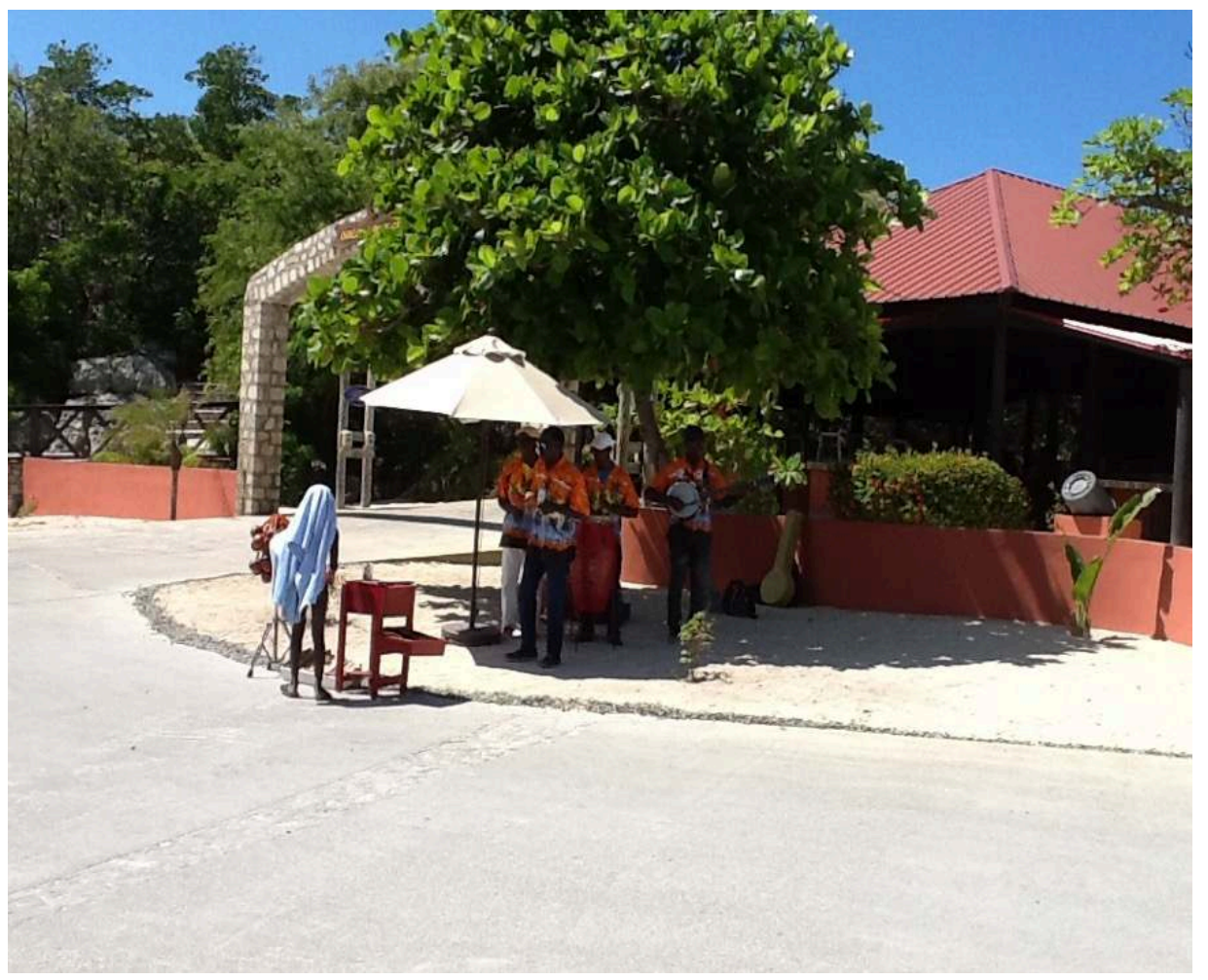




\section{Une appropriation bien acquise en Guadeloupe : l'exemple de Sainte-Anne}

En Guadeloupe, la grande hôtellerie internationale a joué un rôle moteur dans la mise en tourisme. A Sainte-Anne, située en Grande Terre, c'est le Club Méditerranée qui a impulsé le développement territorial basé en partie sur le tourisme. Ce village de pêcheurs a ensuite accueilli des résidences secondaires, des résidents aisés travaillant dans l'agglomération de Pointe-à-Pitre, des locations pour les Guadeloupéens comme pour les touristes métropolitains. Durant les années 1980, les hôtels haut de gamme à capitaux insulaires se sont implantés, entraînant le développement du secteur de la restauration, de la location de voitures et des entreprises qui proposent des excursions en mer autour des îlets de Petite et Grande Terre. En 2017, le front de mer de l'agglomération propose toutes les fonctionnalités d'une station balnéaire.

Figure 3 : Le front de mer, support de l'économie touristique à Sainte-Anne / a) carte localisation
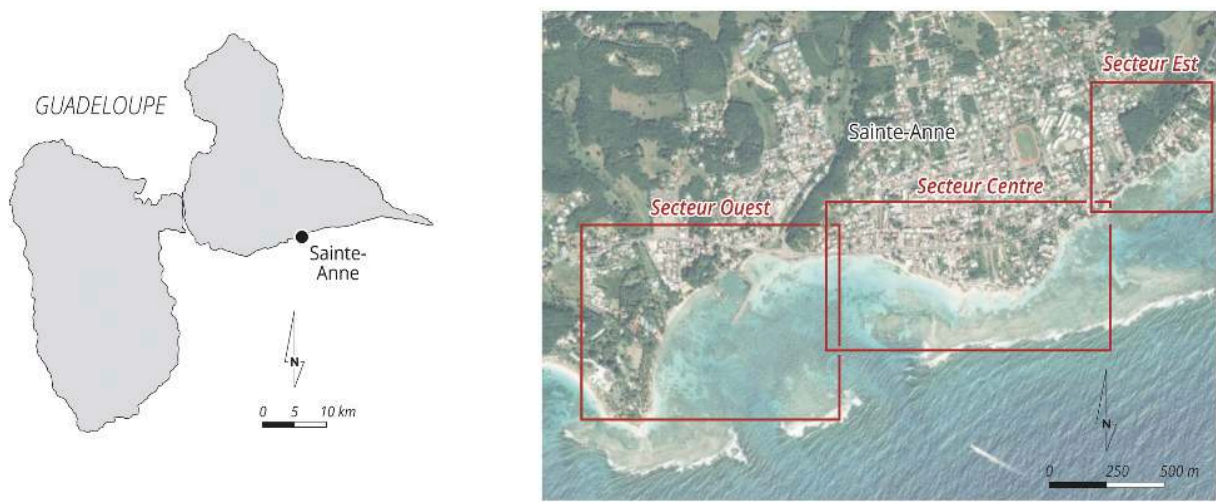
Figure 3 : Le front de mer, support de l'économie touristique à Sainte-Anne / b) secteur Ouest

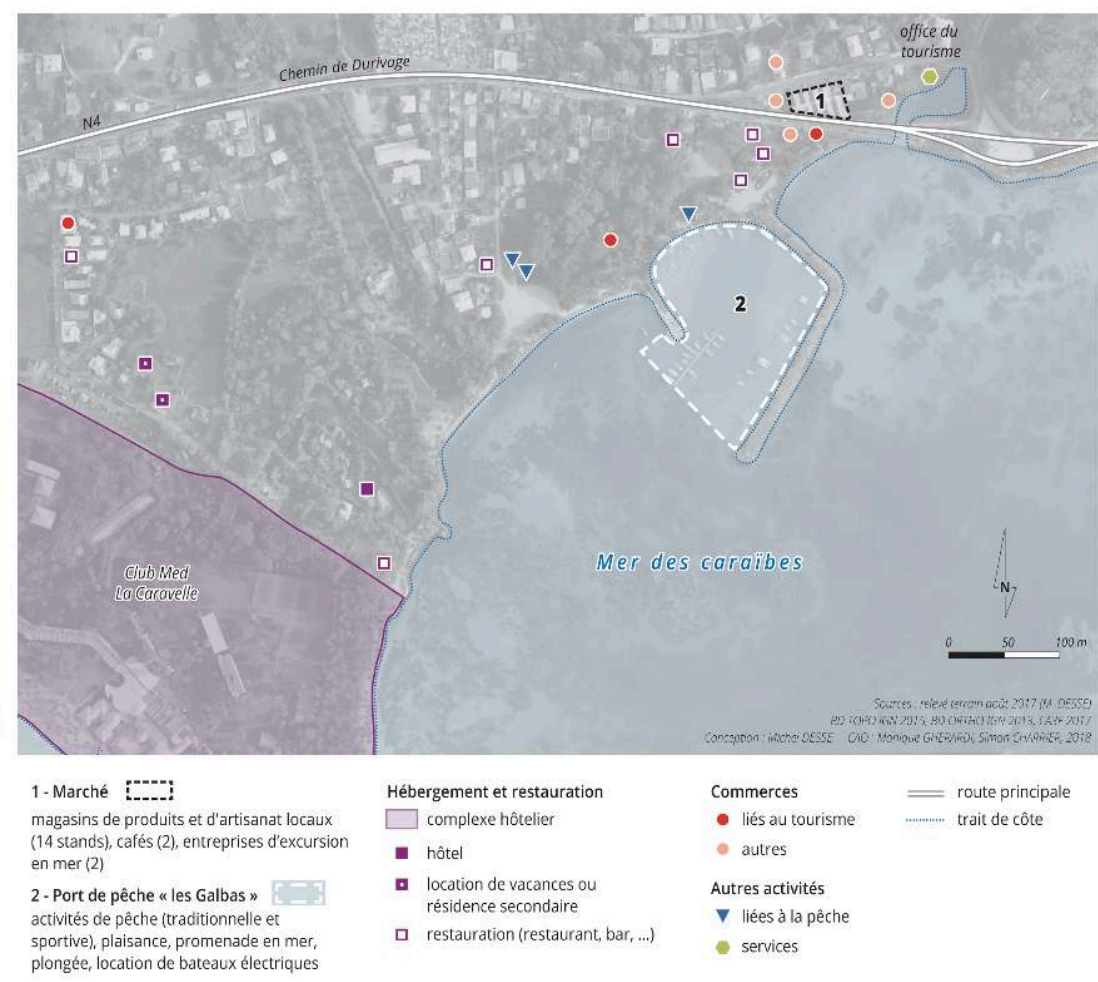

Figure 3 : Le front de mer, support de l'économie touristique à Sainte-Anne / c) secteur Centre

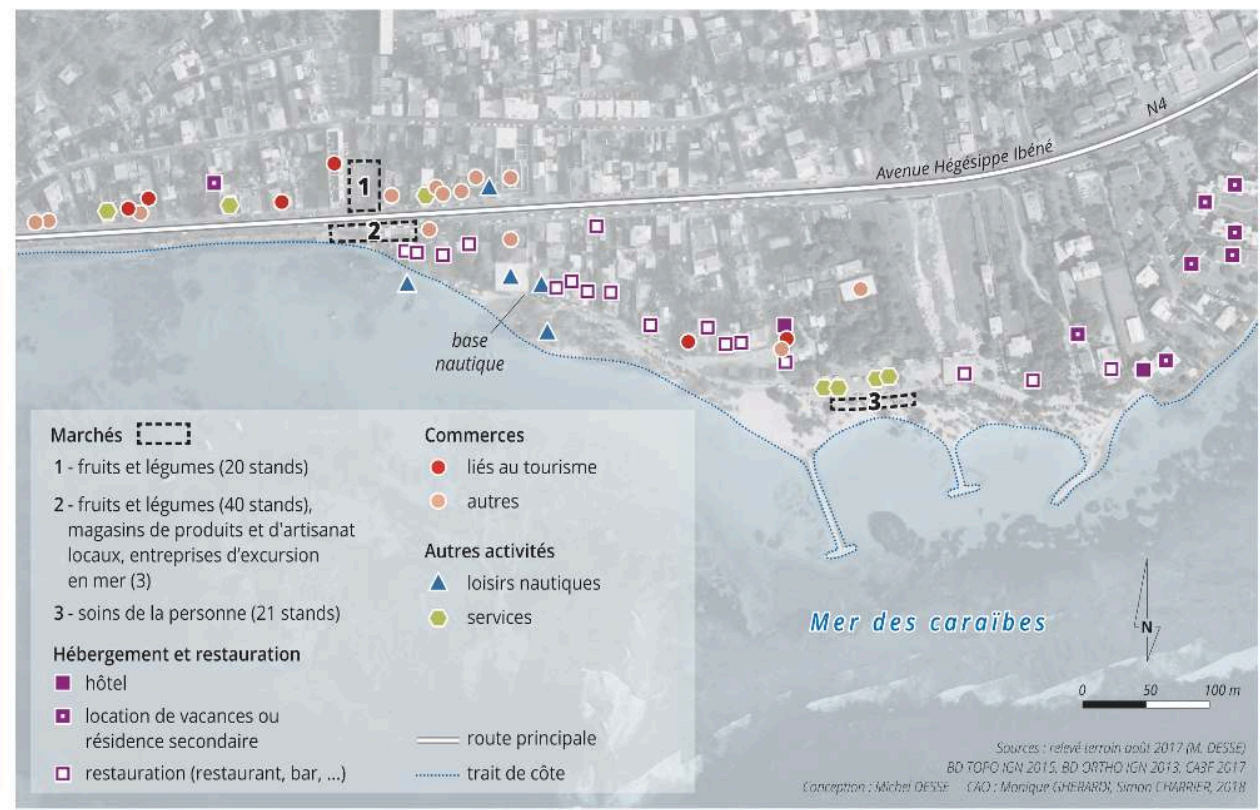


Figure 3 : Le front de mer, support de l'économie touristique à Sainte-Anne / d) secteur Est

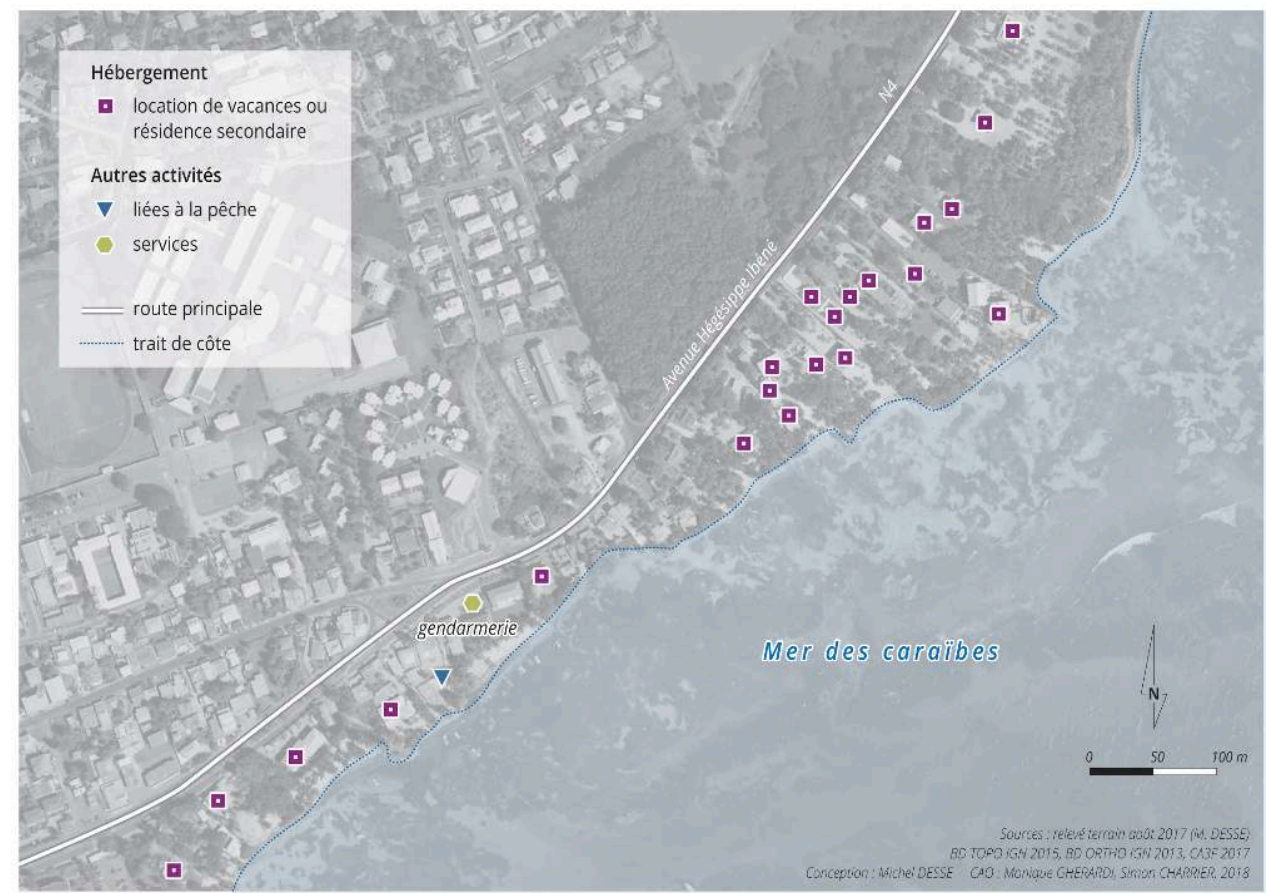

Image 5. La plage de Sainte-Anne, un bord de mer autrefois occupé par les marins pêcheurs, aujourd'hui approprié par les habitants et ouvert au tourisme

Source : Michel Desse, juillet 2017

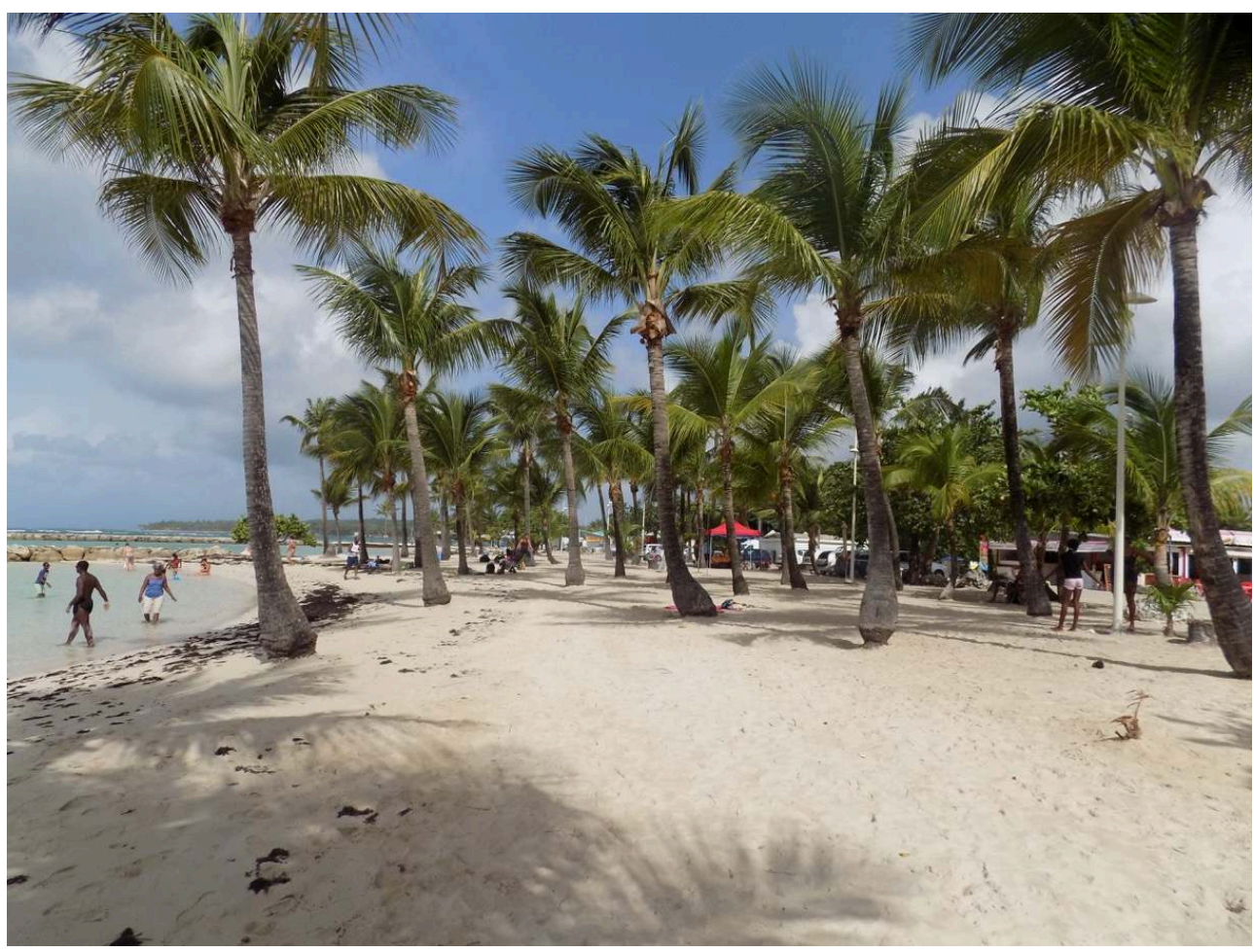

31 Au centre, les plages connaissent une densité des équipements et de fonctions : jeux nautiques, cafés et restaurants de plage, marché artisanal proposant des productions locales et divers services comme les excursions en mer. Un second marché alimentaire, 
accueillant aussi des magasins d'artisanat et de produits locaux, intériorise cette centralité. De part et d'autre, le long du front de mer, les magasins de souvenirs et de vêtements de plage, de restauration rapide remplacent peu à peu l'appareil commercial traditionnel (épicerie) et l'habitat ancien... En effet, les petites cases traditionnelles ne répondent plus aux normes de confort. Ces dernières ont aussi parfois connu des atteintes et des destructions, du fait des houles cycloniques. Un troisième marché artisanal dédié au tourisme assure la jonction entre la zone hôtelière et le bourg. Les activités et les aménagements liés à la pêche, qui constituaient l'essentiel de l'occupation du front de mer, ont été regroupés à la marge du bourg autour d'un petit port accueillant les canots de pêche et les catamarans qui effectuent des sorties en mer. À la zone hôtelière qui occupe l'ouest de la plage répondent les petits hôtels, les entreprises proposant une dizaine de bungalows et les résidences secondaires qui s'étendent en bordure de mer à l'est du bourg. Ponctuellement, en fin de semaine, à l'occasion d'évènements festifs, ces espaces de bord de mer connaissent des afflux de commerces ambulants (tatouages, vente de repas antillais, petit artisanat). Ce passage d'un lieu tourné vers la pêche aux fonctions ludiques et touristiques est pleinement approprié par les habitants, qui y développent toutes les pratiques de plage standard: baignade, natation, aquagym, longe côte, promenade, sans oublier les pratiques locales des déjeuners et des repas familiaux en bord de mer.

Images 6 et 7 Sainte-Anne : le marché en bord de mer, excursion, locations de voitures, artisanat et sur le bord de plage, les bars et les restaurants

Source : Michel Desse, juillet 2017.

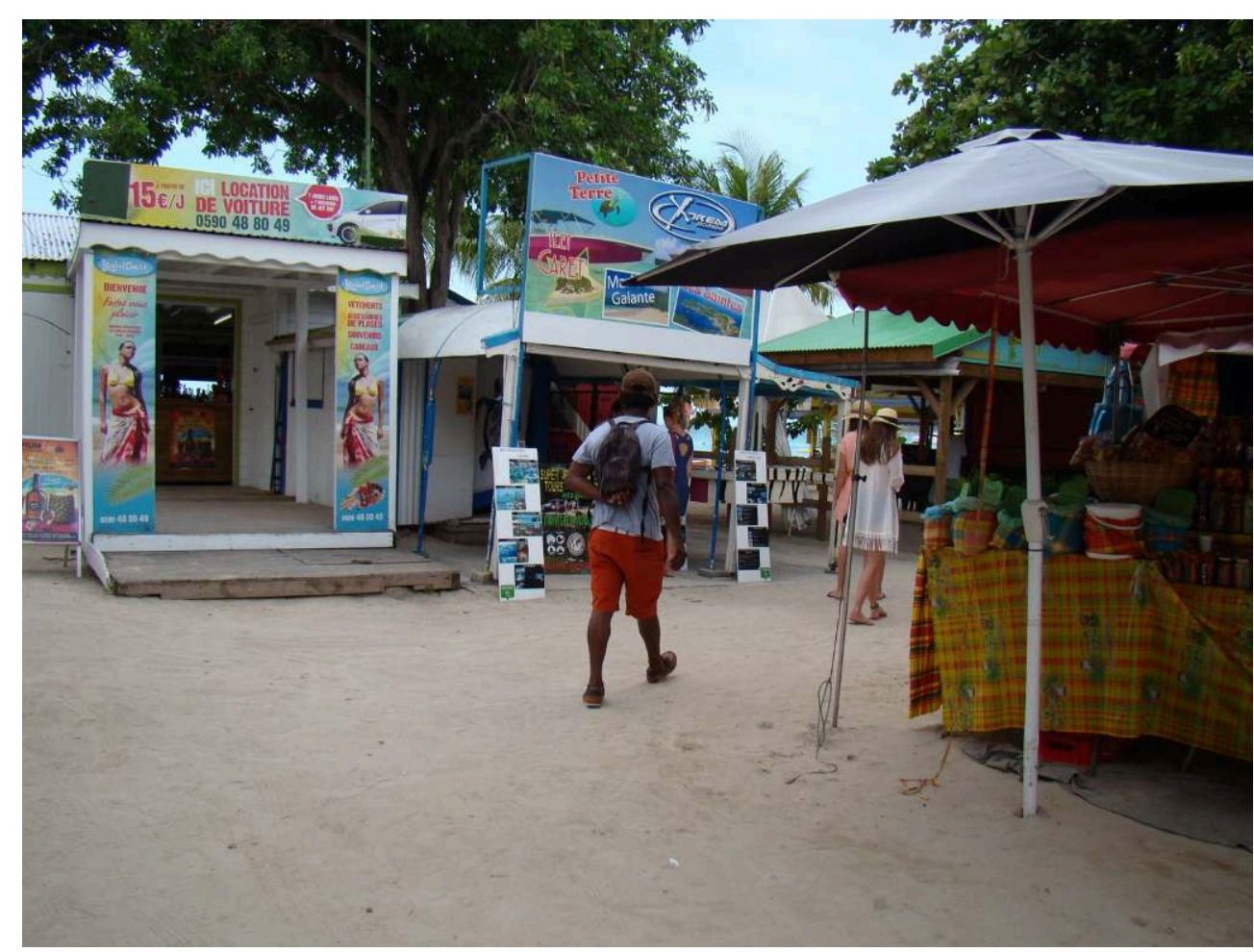




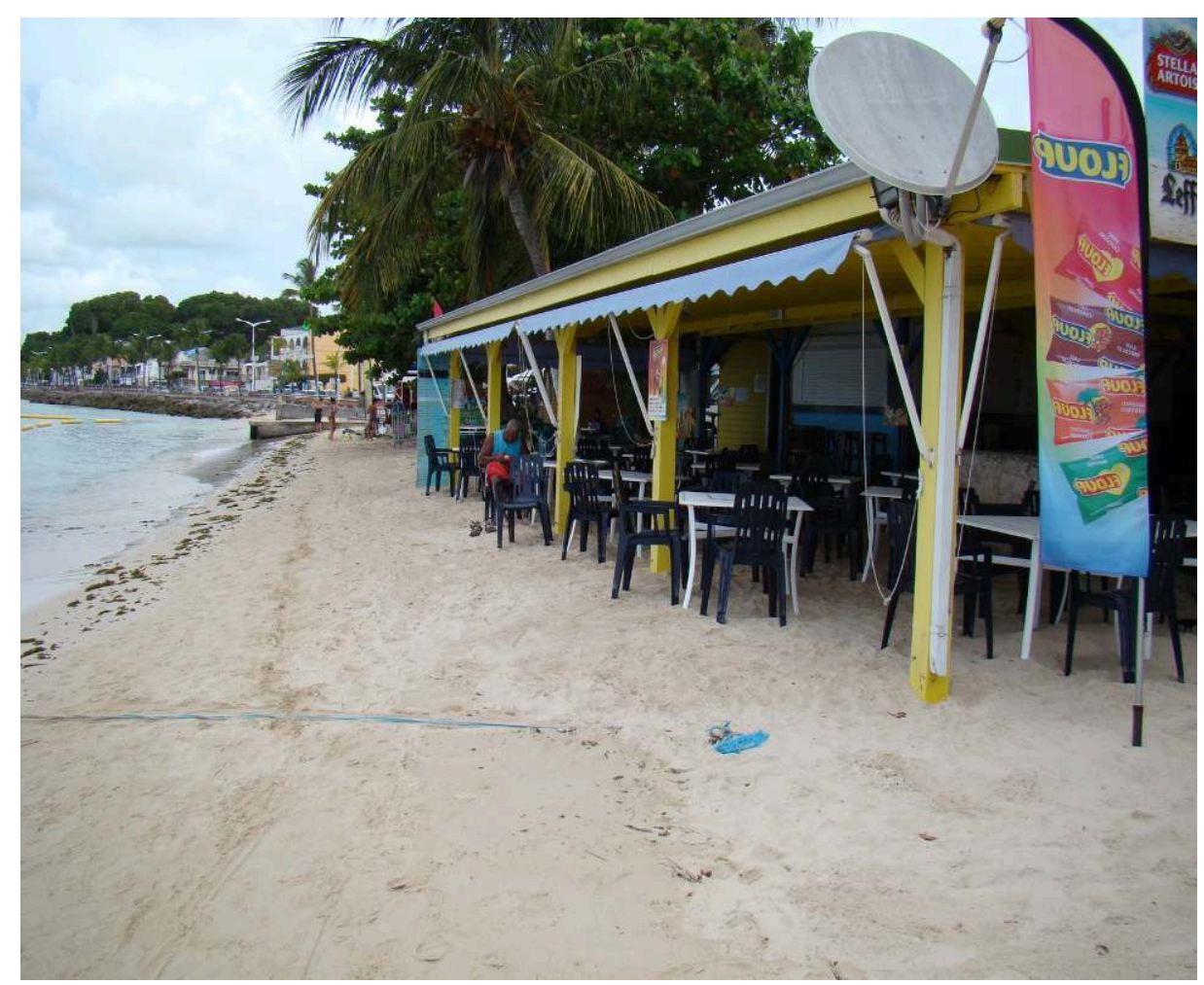

\section{Conclusion}

Depuis près de 50 ans, les îles de la Caraïbe connaissent un essor de l'activité touristique, une progression du nombre de touristes et une large diffusion des zones touristiques - autrement dit, une évolution qui a mené des coquilles hôtelières initiales à de véritables systèmes touristiques incluant des hôtels internationaux (qui coexistent avec la location chez les habitants), des commerces et des services nautiques. Le tourisme a engendré un développement local important et permet aujourd'hui d'accueillir aussi une importante clientèle régionale qui s'ouvre à la fois au tourisme et aux loisirs de plein air, en particulier à toutes les formes de nautisme. On retrouve néanmoins les gradients de la mise en tourisme: lorsque l'activité touristique reste mineure et que les niveaux de développement sont très faibles, comme à Haïti ou dans certaines parties d'îles enclavées, le modèle d'aménagement privilégié demeure celui de l'enclave, qui réduit les contacts entre les populations, les acteurs locaux de la filière touristique et les groupes et clientèles internationales. Dans les îles anciennement ouvertes au tourisme, les acteurs locaux, relayés par des petits investisseurs européens et nord-américains, développent toute la panoplie des activités d'accueil.

Cette dynamique de diffusion est aussi appuyée dans les petites îles par les mesures qui visent à protéger les populations littorales contre les risques de submersion marine liés en partie à l'élévation des niveaux marins. Depuis 1989 et le passage de différents cyclones (Hugo, puis Luis en 1995, Lenny en 1999 et Irma en 2017), les différents acteurs publics profitent des destructions sur la zone littorale - qui relève très souvent du domaine public de l'État - pour éloigner les populations indigentes et les marinspêcheurs du bord de mer. La première ligne de construction est parfois détruite pour recréer les plages et remplacer l'habitat traditionnel par des marchés artisanaux, des 
locations, des petits hôtels, de la restauration de plage et des équipements tournés vers les activités nautiques.

Ainsi, l'économie touristique permet un véritable développement local, répondant aussi à la clientèle insulaire qui trouve un intérêt plus marqué pour les pratiques nautiques récréatives (Nicolas-Bragance F., 2013). Bien entendu, comme tout secteur touristique en milieu insulaire, les équilibres sociétaux, sociaux et environnementaux demeurent fragiles. Une crise économique, sociale ou environnementale, locale ou internationale, peut emporter toute une filière ou la mettre en difficulté. Les crises financières ou géostratégiques ont ainsi touché l'ensemble du secteur touristique durant la décennie 2000. Depuis 2017, c'est en Grande Terre de Guadeloupe que les arrivées d'algues sargasses bordent les plages et comblent les anses, mais aussi les ports, pénalisant les sorties en mer et rendant les bords de mer particulièrement nauséabonds. Enfin, alors que les statistiques tendent à montrer la progression des pratiques touristiques, les différentes îles arriveront-elles à s'ouvrir davantage et à résister à l'ouverture inéluctable de Cuba? Le défi reste majeur.

\section{BIBLIOGRAPHIE}

Burac Maurice, La Barbade. Les mutations récentes d'une île sucrière, Bordeaux, CEGET, coll. « Îles et archipels », 1993.

Burac Maurice, Desse Michel, Les Départements Français d'Amérique à l'aube du XXI siècle : leur développement économique et social (actes de colloque), Paris, Karthala, coll. « Terres d'Amérique », $\mathrm{n}^{\circ} 4,2003$.

Calmont A. et Mérat P.-J., Haïti entre permanences et ruptures. Une géographie du territoire, Ibis Rouge, Guyane Française, 2015.

Chardon Jean-Pierre et Hartog Thierry, « Saint-Barthélemy, un choix et ses limites », Les Cahiers d'Outre-Mer, n 48, Bordeaux, 1995, pp. 176-261.

Chardon Jean-Pierre et Hartog Thierry, « Saint-Martin ou l'implacable logique touristique », Les Cahiers d'Outre-Mer, n 48, Bordeaux, 1995, pp. 21-33.

Connel John, « Tourism in Anguilla », in David Barker, Carol Newby, Mike Morrissey (dir.), A reader in Caribbean geography, Kingston, 1998, pp. 165-171.

Dehoorne Olivier, « La Baie du Marin (Martinique) : l'organisation d'un nouvel espace touristique autour de la plaisance », Études caribéennes [en ligne], n 7, août 2007.

Dehoorne Olivier et Petit-Charles Nathalie, « Tourisme de croisière et industrie de la croisière ", Études caribéennes [en ligne], $\mathrm{n}^{\circ}$ 18, avril 2011.

Desse Michel, «L'inégale maritimité des villes des départements d'Outre-Mer insulaires », in Péron F. (dir.), La maritimité aujourd'hui, Paris, L'Harmattan, coll. « Géographie et Cultures », 1996, pp. 241-249. 
Desse Michel, «L'impact économique de la plaisance à la Martinique », Les Annales de Géographie, $n^{\circ} 613,2000$, pp. 306-316.

Desse Michel, Les fronts de mer : supports urbanistiques et touristiques en Guadeloupe et Martinique, Paris, Karthala, coll. « Terres d'Amérique », n 3, 2001, pp. 343-353.

Desse Michel, Les littoraux de la Caraibe insulaire, des territoires en mutation, Habilitation à Diriger la Recherche, tome 2, Université Paris IV-Sorbonne, 2004.

Desse Michel, Saffache Pascal, Les littoraux antillais : des enjeux de l'aménagement à la gestion durable, Guyane française, Ibis Rouge, 2005.

Desse Michel, «Les territoires du nautisme dans les Petites Antilles », in Le nautisme : acteurs, pratiques et territoires (dossier sous la direction de N. Bernard), Rennes, PUR, 2005, pp. 73-84.

Desse Michel, « La plage : reflet des fractures sociales et ethniques aux Antilles », Études caribéennes, $\mathrm{n}^{\circ} 4,2006$.

Desse Michel, « Le tourisme de croisière, élément refondateur des urbanités antillaises ", in R Knafou et P Violier (dir.), Les mondes urbains du tourisme, Paris, Belin, 2007, pp. 70-283.

Desse Michel, Les îles de la Caraibe, enjeux et perspectives, Paris, L'Harmattan, coll. « Itinéraires géographiques », 2013.

Desse Michel et Nordin Christine, «St-Barthélemy : en rik karibisk ö som utmärker sig », Sverige utanför-Swensk makt och dess spar i utlandet. Svenska Sällskapet för Antropologi och geografi, 2015, pp. 85-102.

Desse Michel et Charrier Simon, «La grande plaisance, un secteur économique en plein essor », Études caribéennes [en ligne], $\mathrm{n}^{\circ}$ 36, avril 2017.

Desse Michel, «Les croisières maritimes dans le monde », in E. Fagnoni (dir.), Les espaces du tourisme et des loisirs, Paris, Armand Colin, 2017, pp. 150-170.

Doura Fred, Économie d'Haïti, dépendance, crises et développement, tome 2, Les éditions Dami, 2012.

Larroque-Chounet Liliane, Les Guadeloupéens et le développement du tourisme, CENADOM, Talence, 1989.

Miossec Jean-Marie, Le tourisme et les aménagements touristiques littoraux, Géographie humaine des littoraux maritimes, SEDES, 1998, pp. 310-412.

Sélise Mario, Dynamiques et recompositions urbaines dans les Petites Antilles, Thèse de géographie, Université des Antilles et de la Guyane, 2010.

Nicolas-Bragance Fabiola, Patrimoine culturel festif et tourisme : une interaction en question. Quelle stratégie pour la Martinique, la Guadeloupe et la Guyane?, Thèse de géographie, Université des Antilles et de la Guyane, 2013.

UNWTO, Faits saillants OMT du tourisme, 2017, 16 pages.

\section{RÉSUMÉS}

Les îles de la Caraïbe constituent une région touristique ancienne et dynamique, mais qui dépend de la conjoncture mondiale. Les différentes formes du tourisme répondent aux attentes d'une clientèle variée et sont plus ou moins appropriées par les acteurs insulaires, permettant le développement local. En général, le niveau de développement induit une appropriation forte et la multiplication des acteurs et des activités en lien avec le tourisme. 
The Caribbean islands constitute a dynamic and old touristic area, but it depends on the international economic context. Different forms of tourism meet the requirements of varied customers and are more or less appropriated by insular actors, allowing local development. The level of development generally leads to a strong appropriation by the populations and a multiplication of activities and actors that work in connection with the touristic sector.

Las islas del Caribe forman una región turística antigua y dinámica pero que continúa dependiendo del contexto mundial. Las diferentes formas de turismo satisfacen las necesidades de una clientela variada, y son más o menos integradas por los actores insulares, lo que permite el desarrollo local. En general, el nivel de desarrollo lleva a una apropiación fuerte y a la multiplicación de los actores y las actividades en relación con el sector del turismo.

\section{INDEX}

Palabras claves : Islas del Caribe, turismo, desarrollo local, turismo de estancia, turismo de crucero, gran navegación de recreo.

Mots-clés : îles de la Caraïbe, tourisme, développement local, tourisme de séjour, tourisme de croisière, grande plaisance.

Keywords : Caribbean islands, tourism, local development, longer stay tourism, cruise tourism, big sailing, pleasure yachting.

\section{AUTEURS}

\section{MICHEL DESSE}

Professeur de géographie à l'Université de Nantes, travaille sur la Caraïbe depuis 1984. Il a publié de nombreux articles et ouvrages. Les îles de la Caraïbe, enjeux et perspectives, est paru chez L'Harmattan, coll. « Itinéraires géographiques » en 2013. Les recherches actuelles portent sur les formes d'adaptation des sociétés littorales aux changements climatiques. michel.desse@univnantes.fr

\section{JUSLINE RODNE JEANTY}

Doctorante en quatrième année à l'Université de Nantes. Le sujet de thèse porte sur la dégradation du littoral du Nord-Est d'Haïti, sous la direction du Professeur Michel Desse. Professeure-chercheure à l'Université d'Etat d'Haïti, Campus Henry Christophe de Limonade. jusline.rodne@univ-nantes.fr // rodnejusline12@yahoo.fr

\section{MONIQUE GHERARDI}

Ingénieur d'études à l'UMR GRED (UPVM3/IRD). Son implication dans plusieurs programmes de recherches l'a emmené sur divers terrains (Martinique, Guadeloupe, Mayotte, Indonésie, Maroc, Algérie, Tanzanie, Vanuatu) afin de récolter, analyser et traiter des bases de données. Son second domaine de compétence est la formation au logiciel ArcGis (Système d'Information Géographique) auprès de Masters du département de géographie de l'Université Paul Valéry Montpellier 3 (UPVM3). monique.gherardi@univ-montp3.fr

\section{SIMON CHARRIER}

Cartographe à l'Institut de Géographie et d'Aménagement Régional de l'Université de Nantes (IGARUN). Il conçoit et réalise des documents cartographiques dans le cadre de projets, 
d'ouvrages ou de publications scientifiques en collaboration avec les enseignants-chercheurs de l'IGARUN avec notamment une publication récente sur l'essor de la grande plaisance (Desse et Charrier, 2017). Il est, par ailleurs, secrétaire d'édition de la revue "Les Cahiers nantais". simon.charrier@univ-nantes.fr 